\title{
Coherent States of Harmonic Oscillator and Generalized Uncertainty Principle
}

\author{
Kourosh Nozari and Tahereh Azizi \\ Department of Physics, Faculty of Basic Science, \\ University of Mazandaran, \\ P. O. Box 47416-1467, Babolsar, IRAN \\ e-mail:knozari@umz.ac.ir
}

\begin{abstract}
In this paper dynamics and quantum mechanical coherent states of a simple harmonic oscillator are considered in the framework of Generalized Uncertainty Principle(GUP). Equations of motion for simple harmonic oscillator are derived and some of their new implications are discussed. Then coherent states of harmonic oscillator in the case of GUP are compared with relative situation in ordinary quantum mechanics. It is shown that in the framework of GUP there is no considerable difference in definition of coherent states relative to ordinary quantum mechanics. But, considering expectation values and variances of some operators, based on quantum gravitational arguments one concludes that although it is possible to have complete coherency and vanishing broadening in usual quantum mechanics, gravitational induced uncertainty destroys complete coherency in quantum gravity and it is not possible to have a monochromatic ray in principle.
\end{abstract}

PACS: 04.60.-m, 11.17.+y

Keywords: Quantum Gravity, Generalized Uncertainty Principle, Quantum Dynamics, Harmonic Oscillator and Coherent States 


\section{Introduction}

Recently it has been indicated that measurements in quantum gravity are governed by generalized uncertainty principle. There are some evidences from string theory and black holes Physics, based on gedanken experiments, which leads some authors to re-examine usual uncertainty principle of Heisenberg[1]. These evidences have origin on the quantum fluctuation of the background spacetime metric. Introducing of this idea has drown attention and many authors considered various problems in the framework of generalized uncertainty principle[2-14]. Such investigations have revealed that actually spacetime is not commutative, gravity is not fundamental interaction, constants of the nature are not constant and notion of locality and position space representation are not satisfied in Planck scale. Therefore, it seems that a re-formulation of quantum theory should be performed to incorporate gravitational effects in planck scale.

The problem of harmonic oscillator in the context of GUP first has been considered by Kempf et al[15]. They have found eigenvalues and eigenfunctions of harmonic oscillator in the context of GUP by direct solving of the Schrödinger equation. Then Camacho has analyzed the role that GUP can play in the quantization of electromagnetic field. He has considered electromagnetic oscillation modes as simple harmonic oscillations[16].

In this paper we first consider the problem of dynamics for harmonic oscillator in the framework of GUP. Then quantum mechanical coherent states of a simple harmonic oscillator are examined in GUP. Some arguments, based on quantum gravitational viewpoint, will show that although there is no difference between the representation and definition of coherent states in the GUP and usual quantum mechanics, quantum gravitational uncertainty will destroy the notion of complete coherency. This will be shown by calculating expectation values of momentum and position operators and their variances. Note that this is a novel implication of GUP since in usual quantum mechanics it is possible to have complete coherency in principle.

The structure of the paper is as follow: section 2 provides an overview to GUP. Section 3 is devoted to dynamics of simple harmonic oscillator in GUP. Section 4 considers quantum mechanical coherent states of harmonic oscillator. The paper follows by summary in section 5 . 


\section{A Generalized Uncertainty Principle}

Usual uncertainty principle of quantum mechanics, the so-called Heisenberg uncertainty principle, should be re-formulated because of the non-commutative nature of space-time. This statement leads to existence of a minimal observable distance on the order of Plank length $l_{P}$ in quantum gravity. In the context of string theories, this observable distance is referred to GUP. A generalized uncertainty principle can be formulated as

$$
\Delta x \geq \frac{\hbar}{\Delta p}+\text { const.G } \Delta p,
$$

which, using the minimal nature of $l_{P}$ can be written as,

$$
\Delta x \geq \frac{\hbar}{\Delta p}+l_{p}^{2} \frac{\Delta p}{\hbar} .
$$

The corresponding Heisenberg commutator now becomes,

$$
[x, p]=i \hbar\left(1+\beta p^{2}\right)
$$

Actually as Kempf et al have argued[15], one can consider more generalization such as

$$
\Delta x \Delta p \geq \frac{\hbar}{2}\left(1+\alpha(\Delta x)^{2}+\beta(\Delta p)^{2}+\gamma\right)
$$

and the corresponding commutator relation is

$$
[x, p]=i \hbar\left(1+\alpha x^{2}+\beta p^{2}\right) .
$$

The main consequence of these GUPs is that measurement of position is possible only up to Plank length, $l_{P}$. So one can not setup a measurement to find more accurate particle position than Plank length.

\section{Dynamics of Harmonic Oscillator in GUP}

For simplicity we consider GUP as equation (3). In Heisenberg picture of quantum mechanics, equation of motion for observable $A$ is as follow,

$$
\frac{d A}{d t}=\frac{i}{\hbar}[H, A] .
$$


Hamiltonian for a simple harmonic oscillator is,

$$
H=\frac{p^{2}}{2 m}+\frac{1}{2} m \omega^{2} x^{2}
$$

Now the equations of motion for $x$ and $p$ are respectively,

$$
\frac{d x}{d t}=\frac{1}{m}\left(p+\beta p^{3}\right)
$$

and

$$
\frac{d p}{d t}=-\frac{1}{2} m \omega^{2}\left(2 x+\beta x p^{2}+\beta p^{2} x\right)
$$

Using Baker-Hausdorff lemma, a lengthy calculation gives the following equations for time evolution of $x$ and $p$ respectively,

$$
\begin{gathered}
x(t)=x(0) \cos \omega t+\frac{p(0)}{m \omega} \sin \omega t \\
+\beta\left[\frac{p^{3}(0)}{m \omega}(\omega t)-\frac{1}{2}\left(p(0) x(0) p(0)+\frac{3}{2}\left[x(0) p^{2}(0)+p^{2}(0) x(0)\right]\right)(\omega t)^{2}\right. \\
-\left(\frac{5}{6} \frac{p^{3}(0)}{m \omega}-\frac{5}{12} m \omega\left[x^{2}(0) p(0)+p(0) x^{2}(0)\right]-\frac{1}{2} m \omega x(0) p(0) x(0)\right)(\omega t)^{3} \\
\left.+\left(\frac{11}{24}\left[x(0) p^{2}(0)+p^{2}(0) x(0)\right]+\frac{5}{12} p(0) x(0) p(0)-\frac{1}{3} m^{2} \omega^{2} x^{3}(0)\right)(\omega t)^{4}\right],
\end{gathered}
$$

and

$$
\begin{gathered}
p(t)=p(0) \cos \omega t-m \omega x(0) \sin \omega t \\
+\beta\left[-\frac{1}{2} m \omega\left[x(0) p^{2}(0)+p^{2}(0) x(0)\right](\omega t)\right. \\
-\left(p^{3}(0)-\frac{1}{4} m^{2} \omega^{2}\left[p(0) x^{2}(0)+x^{2}(0) p(0)+2 x(0) p(0) x(0)\right]\right)(\omega t)^{2} \\
\left.+\left(\frac{2}{3} m \omega\left[x(0) p^{2}(0)+p^{2}(0) x(0)\right]+\frac{1}{2} p(0) x(0) p(0)-\frac{1}{3} m^{3} \omega^{3} x^{3}(0)\right)(\omega t)^{3}\right],
\end{gathered}
$$

where only terms proportional to first order of $\beta$ are considered. It is evident that in the limit of $\beta \rightarrow 0$ one recover the usual results of ordinary quantum mechanics. The term proportional to $\beta$ shows that in the framework of GUP harmonic oscillator is no longer "harmonic" since, now its time evolution is not oscillatory completely. Now for computing expectation values, we need a well-defined physical state. Note that eigenstates of position operators are not physical states because of existence of a minimal length which completely 
destroys the notion of locality. So we should consider a physical state such as $\mid \alpha>$ where $\mid \alpha>$ is for example a momentum space eigenstate[15]. Suppose that $p_{\alpha}(0)=<\alpha|p(0)| \alpha>$ and $x_{\alpha}(0)=<\alpha|x(0)| \alpha>$. Now the expectation value of momentum operator is,

$$
\begin{gathered}
\frac{<\alpha|p(t)| \alpha>}{m}=\frac{p_{\alpha}(0)}{m} \cos \omega t-\omega x_{\alpha}(0) \sin \omega t \\
+\beta\left[-\frac{1}{2} \omega\left(x_{\alpha}(0) p_{\alpha}^{2}(0)+p_{\alpha}^{2}(0) x_{\alpha}(0)\right)(\omega t)\right. \\
-\left(\frac{p_{\alpha}^{3}(0)}{m}-\frac{1}{4} m \omega^{2}\left[p_{\alpha}(0) x_{\alpha}^{2}(0)+x_{\alpha}^{2}(0) p_{\alpha}(0)+2 x_{\alpha}(0) p_{\alpha}(0) x_{\alpha}(0)\right]\right)(\omega t)^{2} \\
\left.+\left(\frac{2}{3} \omega\left[x_{\alpha}(0) p_{\alpha}^{2}(0)+p_{\alpha}^{2}(0) x_{\alpha}(0)\right]+\frac{1}{2 m} p_{\alpha}(0) x_{\alpha}(0) p_{\alpha}(0)-\frac{1}{3} m^{2} \omega^{3} x_{\alpha}^{3}(0)\right)(\omega t)^{3}\right] .
\end{gathered}
$$

This relation shows that there is a complicated dependence of the expectation value of momentum operator to the mass of the oscillator. In usual quantum mechanics, $\frac{\langle\alpha|p(t)| \alpha\rangle}{m}$ and $\frac{p_{\alpha}(0)}{m}$ are mass independent. Here although $\frac{p_{\alpha}(0)}{m}$ is still mass independent, but now $\frac{\langle\alpha|p(t)| \alpha\rangle}{m}$ has a complicated mass dependence. This is a novel implication which have been induced by GUP. Physically, it is completely reasonable that the expectation value for momentum of a particle be a function of its mass, but the mass dependence here has a complicated form relative to usual situation.

\section{Coherent States of Harmonic Oscillator}

We consider the simple harmonic oscillator by hamiltonian

$$
H=\frac{1}{2 m}\left(p^{2}+m^{2} \omega^{2} x^{2}\right)
$$

The problem of quantum oscillator is easily solved in terms of the annihilation and creation operators $a$ and $a^{\dagger}$. We recall the fundamental definitions:

$$
\begin{aligned}
a & =\sqrt{\frac{m \omega}{2 \hbar}}\left(x+\frac{i p}{m \omega}\right), \\
a^{\dagger} & =\sqrt{\frac{m \omega}{2 \hbar}}\left(x-\frac{i p}{m \omega}\right)
\end{aligned}
$$

and the inverse relations:

$$
x=\sqrt{\frac{\hbar}{2 m \omega}}\left(a+a^{\dagger}\right), \quad p=i \sqrt{\frac{m \hbar \omega}{2}}\left(-a+a^{\dagger}\right) .
$$


The Hamiltonian $\mathrm{H}$ is given in terms of these operators as :

$$
H=\hbar \omega\left(a^{\dagger} a+\frac{1}{2}\right)
$$

If we set $N \equiv a^{\dagger} a(:$ Number operator), then

$$
\left[N, a^{\dagger}\right]=a^{\dagger}, \quad[N, a]=-a, \quad\left[a^{\dagger}, a\right]=-1
$$

Let $\mathbf{H}$ be a Fock space generated by $a$ and $a^{\dagger}$, and $\{|n\rangle \mid n \in\{N\} \cup\{0\}\}$ be its basis. The action of $a$ and $a^{\dagger}$ on $\mathbf{H}$ are given by

$$
a|n\rangle=\sqrt{n}|n-1\rangle, \quad a^{\dagger}|n\rangle=\sqrt{n+1}|n+1\rangle, \quad N|n\rangle=n|n\rangle
$$

Where $|0\rangle$ is a normalized vacuum $(a|0\rangle=0$ and $\langle 0 \mid 0\rangle=1)$. Therefore states $|n\rangle$ for $n \geq 1$ are given by

$$
|n\rangle=\frac{a^{\dagger^{n}}}{\sqrt{n !}}|0\rangle
$$

These states satisfy the orthogonality and completeness conditions

$$
\langle m \mid n\rangle=\delta_{m n}, \quad \sum_{n=0}^{\infty}|n\rangle\langle n|=1 .
$$

By definition, coherent state is the normalized state $|\lambda\rangle \in \mathbf{H}$, which is the eigenstate of annihilation operator and satisfies the following equation,

$$
a|\lambda\rangle=\lambda|\lambda\rangle \quad \text { where } \quad\langle\lambda \mid \lambda\rangle=1
$$

and

$$
|\lambda\rangle=e^{-|\lambda|^{2} / 2} \sum_{n=0}^{\infty} \frac{\lambda^{n}}{\sqrt{n !}}|n\rangle=e^{-|\lambda|^{2} / 2} e^{\lambda a^{\dagger}}|0\rangle .
$$

Actually $\lambda$ can be complex because $a$ is not Hermittian. The coherent state was introduced by Schrödinger as the quantum state of the harmonic oscillator which minimizes the uncertainty equally distributed in both position $x$ and momentum $p$. Let us now consider the following possibility, as a generalization for these two operators in GUP,

$$
\begin{aligned}
a & =\frac{1}{\sqrt{2 \hbar \omega}}(\omega x+i[p+f(p)]), \\
a^{\dagger} & =\frac{1}{\sqrt{2 \hbar \omega}}(\omega x-i[p+f(p)]) .
\end{aligned}
$$


Here $f(p)$ is a function that satisfies three conditions, namely: (i) in the limit $\beta \rightarrow 0$ we recover the usual definition for the creation and annihilation operators,(14) and (15); (ii) if $\beta \neq 0$, then we have (3), and; (iii) $\left[a_{\vec{k}}, a_{\vec{k}^{\prime}}^{\dagger}\right]=i \hbar \delta_{\vec{k} \vec{k}^{\prime}}$. It can be shown that the following function satisfies the aforementioned restriction

$$
f\left(p_{\vec{k}}\right)=\sum_{n=1}^{\infty} \frac{(-\beta)^{n}}{2 n+1} p_{\vec{k}}^{2 n+1}
$$

Condition (iii) means that the usual results, in relation with the structure of the Fock space, are valid in our case, for instance, the definition of the occupation number operator, $N_{\vec{k}}=a_{\vec{k}}^{\dagger} a_{\vec{k}}$, the interpretation of $a_{\vec{k}}^{\dagger}$ and $a_{\vec{k}}$ are creation and annihilation operators, respectively, etc. Clearly, the relation between $p_{\vec{k}}, a_{\vec{k}}$ and $a_{\vec{k}}^{\dagger}$ is not linear, and from the Hamiltonian (13) we now deduce that it is not diagonal in the occupation number representation. Let us now consider

$$
f\left(p_{\vec{k}}\right)=-\frac{\beta}{3} p_{\vec{k}}^{3}
$$

In this form we find $p_{\vec{k}}$ as a function of $a_{\vec{k}}$ and $a_{\vec{k}}^{\dagger}$, namely

$$
p_{\vec{k}}=-i \sqrt{\frac{\hbar \omega}{2}}\left(a_{\vec{k}}-a_{\vec{k}}^{\dagger}\right)\left[1-\sqrt{\frac{\hbar \omega \beta}{8}}\left(a_{\vec{k}}-a_{\vec{k}}^{\dagger}\right)\right]
$$

It is clear that, if $\beta=0$ we recover the usual case. Rephrasing the Hamiltonian as a function of the creation and annihilation operators we find:

$$
H=\sum_{\vec{k}} \hbar \omega\left[N_{\vec{k}}+\sqrt{\frac{\hbar \omega \beta}{8}} g\left(a_{\vec{k}}, a_{\vec{k}}^{\dagger}\right)+\beta \frac{(\hbar \omega)^{2}}{16} h\left(a_{\vec{k}}, a_{\vec{k}}^{\dagger}\right)\right]
$$

where functions $g\left(a_{\vec{k}}, a_{\vec{k}}^{\dagger}\right)$ and $h\left(a_{\vec{k}}, a_{\vec{k}}^{\dagger}\right)$ are:

$$
g\left(a_{\vec{k}}, a_{\vec{k}}^{\dagger}\right)=a_{\vec{k}}^{3}-N_{\vec{k}} a_{\vec{k}}-a_{\vec{k}} N_{\vec{k}}-a_{\vec{k}}-\left(a_{\vec{k}}^{\dagger}\right)^{3}+N_{\vec{k}} a_{\vec{k}}^{\dagger}+a_{\vec{k}}^{\dagger} N_{\vec{k}}+a_{\vec{k}}^{\dagger}
$$

and

$$
\begin{gathered}
h\left(a_{\vec{k}}, a_{\vec{k}}^{\dagger}\right)=a_{\vec{k}}^{4}+a_{\vec{k}}^{2}\left(a_{\vec{k}}^{\dagger}\right)^{2}-a_{\vec{k}}^{3} a_{\vec{k}}^{\dagger}-a_{\vec{k}}^{2} a_{\vec{k}}^{\dagger} a_{\vec{k}} \\
+\left(a_{\vec{k}}^{\dagger}\right)^{2} a_{\vec{k}}^{2}+\left(a_{\vec{k}}^{\dagger}\right)^{4}-\left(a_{\vec{k}}^{\dagger}\right)^{2} a_{\vec{k}} a_{\vec{k}}^{\dagger}-\left(a_{\vec{k}}^{\dagger}\right)^{3} a_{\vec{k}} \\
-a_{\vec{k}} a_{\vec{k}}^{\dagger} a_{\vec{k}}^{2}-a_{\vec{k}}\left(a_{\vec{k}}^{\dagger}\right)^{3}+a_{\vec{k}} a_{\vec{k}}^{\dagger} a_{\vec{k}} a_{\vec{k}}^{\dagger}+a_{\vec{k}}\left(a_{\vec{k}}^{\dagger}\right)^{2} a_{\vec{k}} \\
-a_{\vec{k}}^{\dagger} a_{\vec{k}}^{3}-a_{\vec{k}}^{\dagger} a_{\vec{k}}\left(a_{\vec{k}}^{\dagger}\right)^{2}+a_{\vec{k}}^{\dagger} a_{\vec{k}}^{2} a_{\vec{k}}^{\dagger}+a_{\vec{k}}^{\dagger} a_{\vec{k}} a_{\vec{k}}^{\dagger} a_{\vec{k}} .
\end{gathered}
$$


Now with these pre-requisites we can consider the coherent states in the context of GUP. Suppose $|\lambda\rangle$ be an eigenstate of the annihilation operator. We remember that the definition of the annihilation operator in GUP may be different from the usual quantum mechanics but the fact that eigenstates of annihilation operator are coherent states do not changes. Therefore one can write

$$
a|\lambda\rangle=\lambda|\lambda\rangle
$$

Indeed $|n\rangle$ is the eigenstate of the number operator and satisfies completeness and orthogonality conditions. So we can expand $\mid \lambda>$ in terms of the stationary states $|n\rangle$

$$
|\lambda\rangle=\sum_{n=0}^{\infty}|n\rangle\langle n \mid \lambda\rangle=C_{n}|n\rangle,
$$

The eigenvalue equation(19) implies the following recursion formula for the expansion coefficients:

$$
C_{n}=\frac{\lambda}{\sqrt{n}} C_{n-1},
$$

We immediately obtain

$$
C_{n}=\frac{\lambda^{n}}{\sqrt{n !}} C_{0},
$$

The constant $C_{0}$ is determined from the normalization condition on the Fock space,

$$
1=\langle\lambda \mid \lambda\rangle=\left|C_{0}\right|^{2} \sum_{n=0}^{\infty} \frac{\lambda^{2 n}}{\sqrt{n !}}=\left|C_{0}\right|^{2} e^{|\lambda|^{2}},
$$

For any complex number $\lambda$ the correctly normalized quasi-classical state $|\lambda\rangle$ is therefore given by

$$
|\lambda\rangle=e^{-\frac{1}{2}|\lambda|^{2}} \sum \frac{|\lambda|^{n}}{\sqrt{n !}}|n\rangle,
$$

We recall that the $\mathrm{n}$-th stationary state $|n\rangle$ is obtained from the ground state wave function by repeated application of the operator $a^{\dagger}$,

$$
|n\rangle=\frac{1}{\sqrt{n !}}\left(a^{\dagger}\right)^{n}|0\rangle,
$$

This allows us to write the coherent state in the form:

$$
|\lambda\rangle=e^{-\frac{1}{2}|\lambda|^{2}} \sum_{n=0}^{\infty} \frac{1}{n !}\left(\lambda a^{\dagger}\right)^{n}|0\rangle=e^{-\frac{1}{2}|\lambda|^{2}} e^{\lambda a^{\dagger}}|0\rangle
$$


We see that this expression for the eigenstates of the annihilation operator is the same as usual quantum mechanics, equation (23). Actually, it is not surprising that there is no changes in the form of states by modifying the uncertainty relation and similarly for the coherent state. The unchanged state itself cannot be the result of considering generalized uncertainty principle (GUP). It is because a quantum state does not necessarily imply a direct connection with uncertainty principle. Differences caused by different uncertainty relations (such as the GUP) will be found in the expectation values of the operators for a given state and their statistics(such as variance) that can be obtained from the measurement on the state. To analyze the coherent state under the GUP, we should consider $\langle x\rangle$ and $\langle p\rangle$ for the coherent state and see that whether they are changed or not. For this end, suppose that $\mid \lambda>$ is a coherent state given by (39). Since

$$
x=\sqrt{\frac{\hbar}{2 \omega}}\left(a_{\vec{k}}+a_{\vec{k}}^{\dagger}\right)
$$

and

$$
p=-i \sqrt{\frac{\hbar \omega}{2}}\left(a_{\vec{k}}-a_{\vec{k}}^{\dagger}\right)\left[1-\sqrt{\frac{\hbar \omega \beta}{8}}\left(a_{\vec{k}}-a_{\vec{k}}^{\dagger}\right)\right]
$$

one finds the following result for the expectation value of position operator, $x$

$$
<x>=\left\langle\lambda|x| \lambda>=\sqrt{\frac{\hbar}{2 \omega}}<\lambda\left|a_{\vec{k}}+a_{\vec{k}}^{\dagger}\right| \lambda>=\sqrt{\frac{\hbar}{2 \omega}}\left(\lambda+\lambda^{*}\right) .\right.
$$

Therefore one has,

$$
<x>^{2}=\frac{\hbar}{2 \omega}\left(\lambda^{2}+\lambda^{* 2}+2 \lambda \lambda^{*}\right)=\frac{\hbar}{2 \omega}\left(\lambda+\lambda^{*}\right)^{2} .
$$

It is straightforward to show that,

$$
<x^{2}>=\frac{\hbar}{2 \omega}\left(\lambda^{2}+\lambda^{* 2}+2 \lambda \lambda^{*}+1\right)=\frac{\hbar}{2 \omega}\left(\lambda+\lambda^{*}\right)^{2}+1,
$$

and therefore we find for the variance of $x$,

$$
(\Delta x)^{2}=<x^{2}>-<x>^{2}=\frac{\hbar}{2 \omega} .
$$

This is the same as usual quantum mechanics result. This is not surprising since the definition of position operator is the same as its definition in usual quantum mechanics. In the same manner, a simple calculation gives,

$$
<p>=-i \sqrt{\frac{\hbar \omega}{2}}\left[\left(\lambda-\lambda^{*}\right)-\sqrt{\frac{\hbar \omega \beta}{8}}\left[\left(\lambda-\lambda^{*}\right)^{2}-1\right]\right],
$$


and

$$
\begin{gathered}
<p>^{2}=-\frac{\hbar \omega}{2}\left\{\left(\lambda-\lambda^{*}\right)^{2}-2 \sqrt{\frac{\hbar \omega \beta}{8}}\left(\lambda-\lambda^{*}\right)\left[\left(\lambda-\lambda^{*}\right)^{2}-1\right]+\right. \\
\left.\frac{\hbar \omega \beta}{8}\left[\left(\lambda-\lambda^{*}\right)^{2}-1\right]^{2}\right\} .
\end{gathered}
$$

Since,

$$
p^{2}=-\frac{\hbar \omega}{2}\left[\left(a_{\vec{k}}-a_{\vec{k}}^{\dagger}\right)^{2}-2 \sqrt{\frac{\hbar \omega \beta}{8}}\left(a_{\vec{k}}-a_{\vec{k}}^{\dagger}\right)^{3}+\frac{\hbar \omega \beta}{8}\left(a_{\vec{k}}-a_{\vec{k}}^{\dagger}\right)^{4}\right]
$$

then,

$$
\begin{gathered}
<p^{2}>=-\frac{\hbar \omega}{2}\left\{\left[\left(\lambda-\lambda^{*}\right)^{2}-1\right]-\right. \\
2 \sqrt{\frac{\hbar \omega \beta}{8}}\left(\lambda^{3}-\lambda^{* 3}-3 \lambda^{*} \lambda^{2}+3 \lambda^{* 2} \lambda+3 \lambda^{*}-3 \lambda\right)+ \\
\left.\frac{\hbar \omega \beta}{8}\left(\lambda^{4}+\lambda^{* 4}-4 \lambda^{*} \lambda^{3}-4 \lambda^{* 3} \lambda+6 \lambda^{* 2} \lambda^{2}-6 \lambda^{2}-6 \lambda^{* 2}+12 \lambda^{*} \lambda+3\right)\right\},
\end{gathered}
$$

and therefore one finds,

$$
(\Delta p)^{2}=<p^{2}>-<p>^{2}=-\frac{\hbar \omega}{2}\left[-1-2 \sqrt{\frac{\hbar \omega \beta}{8}}\left(2 \lambda^{*}-2 \lambda\right)+\frac{\hbar \omega \beta}{8}\left(-4 \lambda^{2}-4 \lambda^{* 2}+8 \lambda^{*} \lambda+2\right)\right],
$$

or by some manipulations, one obtains the following result for variance of $p$,

$$
(\Delta p)^{2}=\frac{\hbar \omega}{2}+\hbar \omega \sqrt{\frac{\hbar \omega \beta}{2}}\left(\lambda^{*}-\lambda\right)+\frac{\hbar^{2} \omega^{2} \beta}{8}\left[1-2\left(\lambda^{*}-\lambda\right)^{2}\right]
$$

Note that these results give the usual quantum mechanical results when $\beta \longrightarrow 0$. Equations (46) and (51) show that although the definition of coherent states do not changes in GUP, but because of quantum gravitational effect one can not have coherent state in principle. Now there is a considerable departure from very notion of coherency. In usual quantum mechanics one can have complete coherency in principle. One can localize wave pocket in space completely, at least in principle and wave can propagate without broadening in principle. This is evident from $\Delta x \geq \frac{\hbar}{\Delta p}$. In quantum gravity because of gravitational induced uncertainty, one can not localize wave pocket at all and it is impossible to omit broadening. Therefore in quantum gravity one can not have any solitonic states and any wave pocket will be broad at least by gravitational effects. 


\section{Summery}

In this paper, we have obtained the equation of motion for a simple harmonic oscillator in the framework of GUP using Heisenberg picture of quantum mechanics. In fact in this situation the terminology of "simple" is no longer applicable because of nonlinear terms in equations of dynamics. It has been shown that there is a complicated mass dependence of momentum operator expectation value which has considerable departure from usual quantum mechanical result. This is a novel implication of GUP. Also by a simple calculation we have shown that the definition of the coherent states is not different in the framework of GUP with usual quantum mechanics. But there is some considerable difference due to gravitational uncertainty. Because of these uncertainties, in quantum gravity one can not have solitonic states and any wave pocket will be broad by gravitational effects. The main result of our calculations, specially computation of expectation values and variances, is that in the framework of GUP very notion of coherency breaks down.

\section{Acknowledgement}

We would like to appreciate an unknown referee for him valuable comments and careful reading of the manuscript. Actually we are indebted to him in some sense.

\section{References}

[1] G. Veneziano, Europhys. Lett. 2 (1986) 199; D. Amati, M. Ciafaloni, and G. Veneziano, Phys. Lett. B197 (1987) 81; Int. J. Mod. Phys. A3 (1988) 1615; Phys. Lett. B216 (1989) 41; Nucl. Phys. B347 (1990) 530; D. J. Gross and P. F. Mende, Phys. Lett. B197 (1987) 129; Nucl. Phys. B303 (1988) 407; K. Konishi, G. Paffuti, and P. Provero, Phys. Lett. B234 (1990) 276; R. Guida, K. Konishi, and P. Provero, Mod. Phys. Lett. A6 (1991) 1487; L. J. Garay, Int. J. Mod. Phys. A10 (1995) 145.

[2] M. Maggiore, Phys. Lett. B304 (1993) 65, hep-th/9301067 (1993).

[3] C. Castro, hep-th/9512044, preprint (1995).

[4] A. Camacho, qr-qc/0206006, preprint (2002).

[5] P. Chen, astro-ph/0305025, preprint (2003).

[6] S. Capozziello, G. Lambiase, G. Scarpetta, gr-qc/9910017, preprint (1999). 
[7] M. Maggiore, Phys. Rev. D49 (1994) 5182, hep-th/9305163 (1993).

[8] M. Maggiore, Phys. Lett. B319 (1993) 83, hep-th/9309034 (1993).

[9] R. J. Adler, P. Chen, D. I. Santiago, "The Generalized Uncertainty Principle and Black Hole Remnants", Winner of 3rd place in the 2001 Gravity Research Foundation essay competition, (2001), Gen. Rel. Grav. 33 (2001) 2101.

[10] S. Kalyana Rama, hep-th/0107255, preprint (2001).

[11] A. Camacho, arXiv: gr-qc/0303061, preprint, (2003).

[12] P. Chen, R. J. Adler, gr-qc/0205106, preprint, (2002).

[13] F. Scardigli, R. Casadio, hep-th/0307174, preprint,(2003).

[14] A. Camacho, arXiv: gr-qc/0302096, preprint,(2003).

[15] A. Kempf et al, Phys. Rev. D52 (1995) 1108

[16] A. Camacho, arXiv: gr-qc/0305052, preprint,(2003).

A. Camacho, arXiv: gr-qc/0302096, preprint,(2003). 